\title{
Ansiedade Materna: presença de risco ao desenvolvimento infantil e reteste da triagem auditiva neonatal ${ }^{\#}$ \\ Maternal Anxiety: presence of risk to child development and retest of newborn hearing screenin
}

Eliara Pinto Vieira Biaggio**

Themis Maria Kessler***

\begin{abstract}
Resumo
Este estudo teve como objetivo analisar possíveis associações entre risco ao desenvolvimento infantil e estado materno ansioso, bem como a associação entre a ansiedade materna e o conhecimento sobre o reteste da Triagem Auditiva Neonatal (TAN). Foram entrevistadas 85 mães que compareceram ao Programa de TAN para o reteste audiológico de seus bebês, pois eles haviam falhado na primeira TAN e tinha-se uma suspeita inicial de deficiência auditiva. Realizou-se uma entrevista com as mães com o objetivo de avaliar o conhecimento materno sobre o reteste da TAN. Em seguida, para investigar o nível de ansiedade materna antes da realização do reteste da TAN de seus filhos, aplicou-se o Inventário de Ansiedade de Beck. Por último, foram observados e analisados os Índices de Risco ao Desenvolvimento Infantil - IRDIs para a avaliação da interação entre a mãe e o bebê. Os resultados dessa pesquisa demonstraram que a ansiedade materna correlaciona-se diretamente aos Índices de Risco ao Desenvolvimento Infantil, ou seja, nos casos de presença de ansiedade materna, observou-se que estiveram ausentes os IRDIs 1, 2, 4 e 5. Em relação ao conhecimento sobre o reteste da TAN, foi possível observar que existiu associação significativa entre o nível de ansiedade das mães e a falta de conhecimento sobre o reteste da TAN, pois $86,8 \%$ das mães que não sabiam o que era o reteste eram ansiosas. Concluiu-se que existiu uma associação positiva entre a presença de risco ao desenvolvimento infantil e o estado emocional materno ansioso. Além disso, houve predomínio de ansiedade nas mães que não sabiam o que era a o reteste TAN.
\end{abstract}

Palavras-chave: Audição. Ansiedade. Linguagem Infantil. Relações Mãe-Filho. Triagem Neonatal.

\begin{abstract}
This study aimed to examine possible associations between risk to child development and maternal anxious state, as well as the association between maternal anxiety and knowledge about the retest in the Newborn Hearing Screening (NHS). 85 mothers were interviewed who came to the NHS Programme for audiological retest of their babies, because they had failed in the NHS first and had initial suspicion of hearing impairment. It was carried out an interview with the mothers using a questionnaire aiming to evaluate their knowledge about the retest on the NHS. Then, to investigate the level of maternal anxiety before performing the retest on the NHS for their children the Beck Anxiety Inventory was applied. Finally, we observed and analyzed the Indicators of Risk for Child Development - IRDIs to evaluate the interaction between mother and baby. The results of this research demonstrated that maternal anxiety correlates directly to the Indicators of Risk for Child Development, in other words, in the case of presence of maternal anxiety we observed the absence of IRDIs 1, 2, 4 and 5. In relation to the knowledge about the retest of the NHS, it was observed that there exists a significant association between the level of anxiety of mothers and knowledge absence about retest of the NHS, because $86.8 \%$ of mothers who did not know what the retest is were anxious. We conclude that there was a positive association between the presence of risk to child development and maternal emotional state of anxiety. Also, there was a predominance of anxiety in mothers who did not know what is to retest the NHS.
\end{abstract}

Keywords: Hearing. Anxiety. Child Language. Mother-Child Relations. Neonatal Screening.

DOI: $10.15343 / 0104-7809.20143804384391$

\# Pesquisa financiada pela Coordenação de Aperfeiçoamento de Pessoal de Nível Superior (CAPES); n do processo: 97.547.

* Universidade Federal de Santa Maria - UFSM, Santa Maria-RS, Brasil. E-mail: fe.donato@ hotmail.com

** Departamento de Fonoaudiologia da Universidade Federal de Santa Maria - UFSM, Santa Maria-RS, Brasil. E-mail: eliarapv@yahoo.com.br

*** Departamento de Fonoaudiologia da Universidade Federal de Santa Maria - UFSM, Santa Maria-RS. E-mail: tkessler@terra.com.br As autoras declaram não haver conflitos de interesse. 


\section{INTRODUC̣ÃO}

Para que o bebê se desenvolva fisicamente e emocionalmente bem é necessário ser oferecido a ele um ambiente adequado ${ }^{1}$. No entanto, o período puerperal é propenso a crises, por causa das modificações físicas e psicológicas que acompanham as mães ${ }^{2}$. A literatura indica diversas mudanças que surgem com a chegada do bebê, principalmente porque a identidade feminina é modificada ${ }^{3}$. Tal reorganização é importante para que a mulher possa alterar seus investimentos emocionais, seu tempo, sua energia e suas atividades rotineiras depois do parto ${ }^{4}$.

Como os bebês são vulneráveis, pois dependem da qualidade dos cuidados e da sensibilidade materna, o exercício da função parental, sobretudo a materna, pode tornar-se uma relação mais sensível ao estado ansioso ${ }^{5}$. Considera-se que a atitude emocional da mãe serve para orientar os afetos do bebê e conferir qualidade de vida a sua existência ${ }^{6}$.

Nesse sentido, alterações emocionais maternas, tais como a ansiedade puerperal, podem impedir a mãe de se vincular com o seu filho e constituírem um risco para o desenvolvimento da criança. Esses efeitos podem ser visualizados por meio dos Indicadores de Risco ao Desenvolvimento Infantil (IRDIs) ${ }^{7}$, que são eficientes para captar rupturas relacionais na díade mãe / bebê, sendo que evidencia alterações que podem desregular o desenvolvimento do bebê muito precocemente $^{8}$.

Nesse período emocionalmente delicado para a mãe frenteà necessidade de exercer a função materna, o bebê precisa passar por uma série de avaliações da sua saúde, o que pode influenciar de alguma forma seu estado emocional. Entre as avaliações clínicas do recém-nascido, cita-se a Triagem Auditiva Neonatal (TAN), que tem por objetivo identificar as crianças com suspeita de deficiência auditiva ${ }^{9}$.

No contexto TAN, alterações emocionais maternas são amplamente estudadas, e pesquisas que relacionam questões emocionais e a TAN são múltiplas e diversificadas ${ }^{8,9}$. No entanto, na literatura, não foram encontradas pesquisas que relacionem o estado emocional materno e os Índices de Risco ao Desenvolvimento Infantil
(IRDIs) e o reteste da TAN. Sabe-se que a mãe é a pessoa mais próxima do bebê e, por conta disso, é importante identificar suas vivências e conhecimentos frente à reavaliação do bebê na TAN.

Tais questões merecem atenção dos profissionais de saúde, já que podem influenciar (negativa ou positivamente) o vínculo inicial mãe/ bebê e desenvolvimento posterior da criança. Por isso, qualquer evento que represente uma ameaça a essas trocas entre o bebê / criança e seus pais merece atenção especial. Dessa forma, é importante conhecer quais fatores podem influenciar o processo interativo da díade, a fim de que sejam pensadas formas de impedir ou minimizar os impactos de problemas relacionados à interação entre a mãe e o bebê no contexto da reavaliação auditiva do neonato e os reflexos desses fatos no seu desenvolvimento.

Frente a isso, o objetivo deste trabalho foi analisar possíveis associações entre risco ao desenvolvimento infantil e estado materno ansioso, bem como a associação entre a ansiedade materna e o conhecimento sobre o reteste da TAN.

\section{MÉTODO}

Esta pesquisa configurou-se como quantitativa e de caráter transversal e foi realizada no Programa de Triagem Auditiva Neonatal (TAN) do Hospital Universitário de Santa Maria (HUSM), da Universidade Federal de Santa Maria (UFSM), em Santa Maria, Rio Grande do Sul, Brasil, e está inserida no Projeto de Pesquisa: "Suspeita de Deficiência Auditiva: relações entre estado emocional materno e risco ao desenvolvimento infantil". Foi aprovada pela Direção de Ensino, Pesquisa e Extensão (DEPE) do HUSM e pelo Comitê de Ética em Pesquisa (CEP), da UFSM, sob o n 06291712.0.0000.5346.

O Programa de TAN do HUSM é um serviço de referência localizado na região central do Rio Grande do Sul, vinculado ao Sistema Único de Saúde (SUS) e que atende 31 municípios dessa região. Para este estudo, foram entrevistadas 85 mães que compareceram ao Programa de TAN para o reteste audiológico de seus bebês. O critério de inclusão foi o bebê ter apresentado o resultado "falha" na primeira etapa TAN, o 
que indicou uma suspeita inicial de deficiência auditiva. Essas entrevistas ocorreram no período de setembro de 2012 a julho de 2013. A pesquisa foi realizada em uma sala reservada para essa finalidade, no próprio Setor de Fonoaudiologia onde a TAN é realizada.

Inicialmente, realizou-se uma entrevista com as mães utilizando um questionário elaborado para o presente estudo com o objetivo de avaliar o conhecimento materno sobre o reteste da TAN. Em seguida, para investigar o nível de ansiedade materna antes da realização do reteste da TAN de seus filhos, aplicou-se o Inventário de Ansiedade de Beck ${ }^{10}$. Tal inventário é constituído por 21 itens, que são afirmações descritivas de sintomas de ansiedade e que permitem a classificação em níveis de intensidade de ansiedade: mínimo de 0 a 10, leve de 11 a 19, moderado de 20 a 30 e grave de 31 a 63. A escala é apropriada para idades entre 14 e 80 anos e pode ser usada em sujeitos não psiquiátricos. Quanto ao tempo de aplicação, estima-se de 5 a 10 minutos. Esse instrumento de avaliação foi escolhido pela possibilidade de realizar-se uma avaliação ágil, fácil de ser respondida e não cansativa para as mães. Cabe ressaltar que a psicóloga da equipe do Programa de TAN do HUSM foi quem aplicou tal instrumento no grupo estudado.

Por último, foram observados e analisados os Índices de Risco ao Desenvolvimento Infantil $I_{\text {RDIs }}^{7}$ para a avaliação da interação entre a mãe e o bebê no contexto do reteste da TAN. Em sua metodologia, o protocolo IRDI prevê um acompanhamento longitudinal de bebês nos períodos correspondentes a 4 fases: 0 a 4 meses incompletos, 4 a 8 meses incompletos, 8 a 12 meses incompletos e 12 a 18 meses incompletos. No entanto, para este estudo, foram avaliados apenas os cinco primeiros IRDIS, pois todos os bebês estavam na faixa de 0 a 4 meses incompletos. Esse instrumento foi escolhido porque os índices da fase correspondente de 0 a 4 meses captam de modo importante o funcionamento da posição materna e envolvem diretamente a protoconversação inicial da díade.

Os IRDIs correspondentes a essa fase são: 1- Quando a criança chora ou grita, a mãe sabe o que ela quer: esse índice foi observado a partir de situações em que a mãe supunha que o bebê queria algo e se ela conseguia atribuir uma interpretação possível a essa demanda, como, por exemplo, dormir, mudar de posição no colo, fome. 2- A mãe fala com a criança em um estilo particularmente dirigido a ela ("manhês"): nesse índice, foi analisada a interação entre mãe e a criança em termos de sintonia, não só se a mãe falava em "manhês", mas se sua fala era sintonizada às produções do bebê. 3 - A criança reage ao "manhês": nesse item, foi observado se a criança era engajada na protoconversação e, sobretudo, se buscava ativamente tal participação. Como algumas mães, em função do estado emocional depressivo ou ansioso, não conseguiam conversar com seus filhos de modo sintonizado, a pesquisadora buscou fazer isso com os bebês e analisar tal resposta quando isso ocorria. 4- $A$ mãe propõe algo à criança e aguarda sua reação: aqui foi observada a capacidade de a mãe esperar a resposta do bebê, ou seja, de conferir-lhe turno durante a protoconversação. 5- Há troca de olhares entre a criança e a mãe: nesse item foi observada a comunicação visual entre o bebê e a mãe.

A coleta de dados foi realizada antes do reteste do bebê na TAN, após as mães receberem informações sobre os objetivos e a metodologia do estudo. As que concordaram com os procedimentos a serem realizados assinaram o Termo de Consentimento Livre e Esclarecido (TCLE). Aquelas que não concordaram foram excluídas da pesquisa, sendo então realizada apenas a TAN.

Os dados referentes ao presente estudo foram processados e analisados de forma eletrônica a partir da construção de um banco de dados (Excel ${ }^{\circledR}$ 2007) e de um programa de análise específico para o cumprimento dos objetivos da pesquisa, o software Statistical Package for Social Science 15.0 (SPSS).

A partir desse procedimento, foi utilizado o Teste qui-Quadrado, quando os pressupostos foram atendidos. Quando isso não acontecia, era aplicado o Teste Exato de Fisher para as variáveis que eram pertinentes. Após essa investigação, as variáveis que estavam associadas ao resultado do teste eram avaliadas pela análise de resíduos para identificar quais classes eram associadas. Foram utilizados intervalos de confiança de 95\%, considerando-se $p<0,05$. 


\section{RESULTADOS}

A primeira análise realizada foi a relação entre a ansiedade materna e os Índices de Risco ao Desenvolvimento Infantil (IRDIs), com o objetivo de investigar a associação entre essas variáveis. Tal análise pode ser observada na Tabela 1.

Tabela 1. Relação entre a ansiedade materna e os Índices de Risco ao Desenvolvimento Infantil - IRDIs $(\mathrm{N}=85)$

\begin{tabular}{|c|c|c|c|}
\hline \multirow[t]{2}{*}{ Variáveis } & \multicolumn{2}{|c|}{ Ansiedade Materna } & \multirow[b]{2}{*}{ p - valor } \\
\hline & $\begin{array}{c}\text { Sem alteração } \\
N: 47\end{array}$ & $\begin{array}{c}\text { Com alteração } \\
\text { N: } 38\end{array}$ & \\
\hline \multicolumn{4}{|c|}{ IRDI 1: Quando a criança chora ou grita, a mãe sabe o que ela quer? } \\
\hline Ausente & $0(0,00 \%)$ & $37(97,4 \%)$ & \multirow{2}{*}{$<0,0001^{*}$} \\
\hline Presente & $47(100,0 \%)$ & $1(2,6 \%)$ & \\
\hline \multicolumn{4}{|c|}{ IRDI 2: A mãe fala com a criança num estilo particular ("manhês") } \\
\hline Ausente & $0(0,00 \%)$ & $37(97,4 \%)$ & \multirow{2}{*}{$<0,0001^{*}$} \\
\hline Presente & $47(100,0 \%)$ & $1(2,6 \%)$ & \\
\hline \multicolumn{4}{|c|}{ IRDI 3: A criança reage ao "manhês" } \\
\hline Ausente & $0(0,00 \%)$ & $1(2,3 \%)$ & \multirow{2}{*}{0,447} \\
\hline Presente & $47(100,0 \%)$ & $37(97,7 \%)$ & \\
\hline \multicolumn{4}{|c|}{ IRDI 4: A mãe propõe algo à criança e aguarda sua reação } \\
\hline Ausente & $0(0,00 \%)$ & $37(97,4 \%)$ & \multirow{2}{*}{$<0,0001^{*}$} \\
\hline Presente & $47(100,0 \%)$ & $1(2,6 \%)$ & \\
\hline \multicolumn{4}{|c|}{ IRDI 5: Há trocas de olhares entre a criança e a mãe } \\
\hline Ausente & $0(0,00 \%)$ & $13(34,2 \%)$ & \multirow{2}{*}{$<0,0001^{*}$} \\
\hline Presente & $47(100,0 \%)$ & $25(65,8 \%)$ & \\
\hline
\end{tabular}

Legenda: *Valores estatisticamente significantes $(\mathrm{p}<0,05)$; Teste qui-Quadrado; $\mathrm{N}=$ número de sujeitos avaliados; IRDIs = Índices de Risco ao Desenvolvimento Infantil.

Na Tabela 2, verifica-se a frequência de IRDIs alterados nas mães que apresentaram níveis de ansiedade, ou seja, as mães foram consideradas ansiosas na amostra investigada. Observa-se que os IRDIs mais ausentes foram o IRDI 1, 2 e 4.

Tabela 2. Frequência de alteração de IRDIs em mães ansiosas

\begin{tabular}{|l|c|}
\hline IRDIs & ANSIEDADE \\
\hline IRDI 1: Quando a criança chora ou grita, a mãe sabe o que ela quer? & $37(97,36 \%)$ \\
\hline IRDI 2: A mãe fala com a criança num estilo particular ("manhês") & $37(97,36 \%)$ \\
\hline IRDI 3: A criança reage ao "manhês" & $1(2,64 \%)$ \\
\hline IRDI 4: A mãe propõe algo à criança e aguarda sua reação & $37(97,36 \%)$ \\
\hline IRDI 5: Há trocas de olhares entre a criança e a mãe & $13(7,7 \%)$ \\
\hline TOTAL DE MÃES ANSIOSAS & 38 \\
\hline
\end{tabular}


Finalmente, estudou-se a associação entre o conhecimento materno prévio sobre o que era o reteste da TAN e o nível de ansiedade dessas mães (Tabela 3). Analisando o resultado da Tabela 3, pode-se observar que existe associação significativa entre o nível de ansiedade das mães e a variável que avalia seu conhecimento sobre o reteste da TAN. A associação é dada entre as mães sem alteração de níveis de ansiedade e que sabiam o que era o reteste da TAN. Da mesma forma, a manifestação de algum grau de ansiedade por parte das mães está relacionada a não saber o que é o reteste da TAN.

Tabela 3. Relação entre a ansiedade das mães e o conhecimento sobre o reteste da TAN

\begin{tabular}{|c|c|c|c|}
\hline \multirow[t]{2}{*}{ Variáveis } & \multicolumn{2}{|c|}{ Nível de ansiedade materna } & \multirow{2}{*}{ p - valor } \\
\hline & Sem alteração & Com alteração & \\
\hline \multicolumn{4}{|c|}{ Vocês sabia o que era o reteste da TAN? } \\
\hline Sim & $39(83 \%)$ & $5(13,2 \%)$ & \multirow{2}{*}{$<0,0001^{*}$} \\
\hline Não & $8(17 \%)$ & $33(86,8 \%)$ & \\
\hline
\end{tabular}

Legenda: *Valores estatisticamente significantes $(p<0,05)$; Teste qui-Quadrado.

\section{DISCUSSÃo}

Os resultados dessa pesquisa demonstraram que a ansiedade materna correlaciona-se de modo positivo à presença de índices de risco ao desenvolvimento infantil, ou seja, os bebês dessas mães apresentam risco maior para o seu desenvolvimento do que bebês de mães não ansiosas.

Em relação aos IRDIs, nos casos de presença de ansiedade materna, pode-se observar que estiveram ausentes o IRDI 1 (Quando a criança chora ou grita, a mãe sabe o que ela quer), o IRDI 2 (A mãe fala com a criança num estilo particular), o IRDI 4 (A mãe propõe algo à criança e aguarda sua reação) e o IRDI 5 (Há trocas de olhares entre a criança e a mãe) (Tabela 1).

A ausência do IRDI 1 representa dificuldades das mães estabelecerem demandas de seus bebês. Ressalta-se que essas mães apresentaram níveis de ansiedade alterados, o que pode estar impedindo-as de reconhecer e traduzir as ações da criança, o que implica ausência desse índice. A mãe poderá estar dispersa ou irritada e, muitas vezes, não irá conseguir supor algo que o bebê queira $^{5}$. Nesse caso, a comunicação entre ambos poderá estar comprometida e, consequentemente, a interação prejudicada, pois a mãe pode diminuir sua sensibilidade frente às necessidades do bebêê11,12.
Quanto ao IRDI 2, analisa-se se a mãe fala com a criança num estilo particularmente dirigido a ela ("manhês"), ou seja, esse índice avalia se a mãe interage com o bebê de um jeito particular e em sintonia com as produções do bebê. Frente a isso, nos casos de mães ansiosas, sugere-se que possivelmente elas apresentem dificuldades no investimento dialógico com o bebê, caracterizado pela ausência de uma fala particular dirigida ao fiIho. Devido a ansiedade, as mães podem demonstrar pouco interesse nas produções linguísticas do bebê e acabam perdendo o foco nas produções da criança ${ }^{5}$. Para Beltrami ${ }^{11}$ e Crestani, et al ${ }^{12}$, falhas nesse mecanismo podem facilitar a quebra nessa relação inicial, não sendo suficiente para convocar e atrair o bebê a responder à mãe posteriormente.

Na Tabela 1, nota-se que o IRDI 3 não apresentou relação estatística significativa com a ansiedade materna, pois esse índice esteve presente na maioria dos bebês. No entanto, nos casos de ansiedade materna, a pesquisadora buscou investigar se o bebê se engajava na protoconversação e se buscava ativamente tal participação. Portanto, foram computados os índices conforme esse fato. Nas situações em que as mães não conseguiam conversar com seus filhos de modo sintonizado devido a níveis de ansiedade alterados, a pesquisadora procurou fazer isso e analisar tal resposta. Esse fato permitiu observar que todos os bebês da amostra aqui analisada eram capazes de responder e reagir ao "manhês". 
Outro índice que se mostrou estatisticamente associado com a ansiedade materna (Tabela 1) foi o IRDI 4. A associação está entre as mães com alteração emocional e ausência do IRDI 4, isto é, as mães ansiosas manifestaram ausência desse indicador. Esse índice avalia se a mãe confere turno ao seu filho durante a protoconversação. A mãe precisa convocar seu filho à troca prazerosa e oferecer espaço para o bebê. No entanto, mães com níveis alterados de ansiedade podem não conseguir aguardar a resposta do bebêt . $^{2}$

Uma ruptura nesse processo, seja por condições do bebê ou da mãe, pode levar a um processo de desapropriação da experiência enunciativa inicial do bebê ${ }^{13}$. Essa associação pode favorecer que o bebê torne-se silencioso, pois na medida em que a mãe não propõe algo ao filho e nem oferece sentido as suas vocalizações, o bebê pode não chamar e se fazer chamar ${ }^{14}$.

Para o IRDI 5, a associação estatística está entre alterações emocionais e a ausência desse índice, isto é, as mães ansiosas alteraram esse índice (Tabela 1). Esse resultado demonstra que a ansiedade tende a dificultar as trocas da mãe com o bebê de um modo geral, tanto em níveis de diálogo, quanto em relação às trocas de olhares ${ }^{5}$. O único meio de comunicação entre a mãe e a criança é a expressão emocional e o comportamento que lhe acompanha. Por isso, o destino a ser seguido para o pleno desenvolvimento infantil será determinado pela sua interação e o contexto em que vive, especialmente pela forma que os pais e as mães ou cuidadores primários o tratam e como ele responde a seus genitores ${ }^{15,16}$, que no contexto da ansiedade parece estar prejudicado.

Outro aspecto interessante que cabe uma descrição para reflexão acerca dessa associação entre IRDIS e ansiedade materna é a frequência de alteração desses índices. Na Tabela 2, nota-se que a frequência de alteração dos IRDIS em mães ansiosas são os índices 1, 2 e 4. Corroborando com tal resultado, o estudo de Flores, Beltrami e Souza ${ }^{14}$ apontam que os IRDIs são capazes de captar as interferências de estados emocionais maternos no desenvolvimento infantil e, além disso, as autoras destacam que, dos cinco IRDIs do protocolo, os IRDIs 1, 2 e 4 se relacionam diretamente a estados emocionais maternos, tanto em casos de depressão, como em casos de ansiedade.

No presente trabalho, pode- se notar que, na presença de ansiedade materna, todos os índices estiveram alterados, mas com maior frequência àqueles relacionados essencialmente as iniciativas maternas (IRDI 1, 2 e 4). Além disso, o IRDI 3 e IRDI 5, que se referem à relação entre a díade mãe / bebê, mostram-se frequentemente mais presentes na população estudada (Tabela 2). O IRDI 3 refere-se à reação do bebê a voz, no entanto, quando ele não respondia, a pesquisadora interagia com a criança, que respondia, evidenciando que as falhas interacionais possivelmente acontecem na relação mãe-filho.

Beltrami, Moraes e Souza ${ }^{5}$ também encontraram associação entre índices de risco ao desenvolvimento infantil e estado emocional materno ansioso em bebês que passaram na primeira etapa da TAN. No entanto, ressalta-se que as díades mãe / bebê da presente pesquisa estão passando pelo reteste da TAN, pois haviam falhado na primeira etapa da TAN e apontou-se uma suspeita inicial do déficit sensorial auditivo. $\mathrm{O}$ momento do reteste da TAN coincide com a ocasião em que os pais estão buscando suas identidades e o novo papel que irão desempenhar na estrutura familiar com a chegada do novo membro. Além disso, as emoções estão focalizadas no estabelecimento do vínculo entre os pais e a criança ${ }^{16}$, e, quando ocorre a falha na TAN, a mãe pode vivenciar um período que potencializa a ansiedade, pois a criança precisa realizar novos exames audiológicos para a confirmação ou não do diagnóstico de deficiência auditiva.

Frente a isso, nos últimos anos, alguns pesquisadores têm se voltado para o estudo das atitudes dos familiares diante da TAN. Para Tochetto ${ }^{8}$, entre os principais empecilhos para o sucesso dos programas de TAN estão a falta de informação dos pais e a ansiedade desencadeada nas mães pela situação de seu filho estar sendo testado. Mahl ${ }^{17}$ verificaram que existe tal associação porque ocorre o predomínio de reações negativas frente à testagem do bebê quando as mães não sabem o que é a TAN, sendo que, nesse contexto, os sentimentos mais frequentes são a ansiedade $(50 \%)$, a preocupação $(18,29 \%)$ e medo $(13,41 \%)$ em relação à saúde auditiva do bebê na sua primeira avaliação da TAN.

Analisando o resultado da Tabela 3, pode-se observar que existe associação significativa entre o nível de ansiedade das mães e a variável que avalia seu conhecimento sobre o reteste da TAN. 
A associação é dada entre as mães sem alteração de níveis de ansiedade e que sabiam o que era o reteste da TAN. Da mesma forma, a manifestação de algum grau de ansiedade por parte das mães está relacionada a não saber o que é o reteste da TAN.

Conforme o resultado apresentado na Tabela 3, nota-se que a ansiedade materna está associada à falta de informação sobre a triagem também no reteste da TAN e não somente na primeira testagem do bebê ${ }^{17}$. Para Tochetto ${ }^{8}$, quando as informações recebidas pelos pais são consideradas suficientes, existe o predomínio de sentimentos positivos durante a realização do exame. Já informações insuficientes podem fazer com que os pais reajam de uma forma insatisfatória, ou mesmo apresentem um aumento do grau de preocupação e ansiedade.

Frente a isso, vários estudos sugerem que quando se trata da saúde do bebê deve-se garantir a informação adequada sobre a TAN e sobre a audição do neonato desde a gestação, para diminuir as dúvidas e o grau de preocupações maternas, entre elas a ansiedade, depois do nascimento do bebê. Sabe-se que o acompanhamento durante a gravidez é importante para o desenvolvimento de ações educativas em saúde, além de favorecer a detecção precoce e a prevenção de diversas doenças. Acrescenta-se, ainda, que, durante o pré-natal, geralmente, a família é mais receptiva às sugestões e orientações direcionadas à saúde da criança ${ }^{18,19}$.

Contudo, pode-se dizer que questões relativas à saúde auditiva, ou pelo menos a informação sobre a importância de realizar a TAN, são ainda pouco abordadas durante o pré-natal. Esse dado é salientado nos estudos de Hilú e Zeigelboim ${ }^{18}$ e Ribeiro e Mitre $^{20}$, nos quais a grande maioria das mães entrevistadas, $75 \%$ e $72 \%$, respectivamente, afirmaram não conhecer o teste da TAN. Além disso, o estudo de Mahl ${ }^{17}$ concluiu que a maioria das mães que compareceram a um Programa de TAN tinham sido informadas sobre o teste durante a internação hospitalar e que esse fato se reflete na média de tempo transcorrido entre o nascimento até a realização do exame, que foi entre 31 e 90 dias. Desse modo, as autoras sugerem a necessidade de aumentar a divulgação da TAN no período pré-natal, assegurando que as avaliações possam ser realizadas até os três primeiros meses, favorecendo, assim, a intervenção precoce até os seis meses de idade ${ }^{21}$.

Com isso, as famílias compareceriam à TAN com algum conhecimento prévio sobre o que seria realizado e a importância de um diagnóstico precoce da deficiência auditiva infantil. Assim seria possível viabilizar suporte à família dentro do contexto do programa de TAN, bem como disseminar informações adequadas para cada etapa do processo das avaliações auditivas sobre a TAN. A relevância da TAN ainda é um assunto pouco conhecido pela população em geral. Portanto, é necessário difundir a necessidade da identificação precoce da deficiência auditiva, pois se sabe que, nos primeiros anos de vida, o contato da criança com o mundo sonoro e sua interação com o outro garantirá o aprendizado dos sons da fala, além de desenvolver e organizar seus pensamentos e sentimentos ${ }^{22}$.

\section{CONCLUSÃO}

Os resultados dessa pesquisa demonstram que há uma associação positiva entre presença de risco ao desenvolvimento infantil e estado emocional materno ansioso, bem como ansiedade materna e o não conhecimento acerca do reteste da TAN.

Dessa maneira, pode-se dizer que o risco ao desenvolvimento infantil abrange aspectos referentes ao ambiente onde o bebê vive e ao estado emocional materno, fatos que foram possíveis de serem vistos nesse trabalho por meio das alterações dos IRDIs e pelo estado emocional materno ansioso. Nesse contexto, conclui-se que o comportamento materno se reflete em alterações nos IRDIS, podendo ocorrer dificuldades maternas em atribuir uma interpretação possível às demandas do bebê (IRDI 1), ausência de protoconversação inicial ou, até mesmo, fala materna não sintonizada às produções do bebê (IRDI 2) e problemas em proporcionar trocas de turnos durante a interação com o bebê (IRDI 4). Destaca-se que todos esses índices dependem diretamente de iniciativas maternas, portanto a mãe precisa estar saudável para conseguir perceber as demandas de seu bebê, sendo que, notadamente, as interações iniciais parecem ser suscetíveis em particular ao ambiente em que o bebê vive.

Nesse estudo, também foi possível concluir que a grande maioria das mães entrevistadas não sabia o que era a reavaliação auditiva e houve predomínio de ansiedade nas mães que não sabiam o que era a o reteste TAN. A relevância da triagem auditiva neonatal ainda é um assunto pouco conhecido pela população em geral, inclusive o reteste da TAN. Portanto, é necessário difundir conhecimentos a 
respeito, em benefício de um número cada vez maior de crianças com deficiência auditiva e suas famílias.

Frente a esses fatos, pode-se concluir que a ansiedade materna é multifatorial e pode ser decorrente de aspectos relacionados a problemáticas pessoais que afetam a maternidade em algumas mulheres e que podem trazer risco ao desenvolvimento do bebê ou até mesmo referentes à situação da reavaliação auditiva do bebê no reteste da TAN. Nesse momento de reavaliações auditivas do bebê, podem ser potencializados sentimentos ansiosos na mãe, pois o neonato precisa realizar novos exames audiológicos para a confirmação ou não do diagnóstico de deficiência auditiva.

\section{REFERÊNCIAS}

1. Winnicott DW. Da pediatria à psicanálise. Rio de Janeiro: Imago; 2000.

2. Maldonado MT. Psicologia da gravidez, parto e puerpério. 6a ed. Petrópolis (RJ): Vozes; 1998.

3. Stern DN. A constelação da maternidade: o panorama da psicoterapia pais/bebê. Porto Alegre: Artes Médicas; 1997.

4. Klaus MH, Kennel JH, Klaus PH. Vínculo: construindo as bases para um apego seguro e para a independência. Porto Alegre: Artes Médicas; 2000.

5. Beltrami L, Moraes AB, Souza APR. Ansiedade materna puerperal e risco para o desenvolvimento infantil. Distúrb Comum. 2013;15(2):229-39

6. Böing E, Crepaldi MA. Os efeitos do abandono para o desenvolvimento psicológico de bebês e a maternagem como fator de proteção. Est Psicol. 2004;21(3):211-26. DOI: http://dx.doi.org/10.1590/S0103-166X2004000300006.

7. Lerner R, Kupfer MCM. Psicanálise com crianças: clínica e pesquisa. 1a ed. São Paulo: Escuta; 2008.

8. Tochetto TM, Petry T, Gonçalves MS, Silva ML, Pedroso FS. Sentimentos manifestados por mães frente à triagem auditiva neonatal. Rev CEFAC. 2008;10(4):566-71. DOI: http://dx.doi.org/10.1590/S1516-18462008000400017.

9. Lima MLLT, Assis ABR, Mercês GB, Barros PF, Griz SMS. Triagem auditiva: perfil socioeconômico de mãe. Rev CEFAC. 2008;10(2):254-60. DOI: http://dx.doi.org/10.1590/S1516-18462008000200016.

10. Beck AT, Steer RA. Beck depression inventory manual. San Antonio: Psychological Corporation; 1993.

11. Beltrami L. Ansiedade materna puerperal e risco para alterações no desenvolvimento infantil [dissertação]. Santa Maria (RS): Universidade Federal de Santa Maria; 2011.

12. Crestani AH, Rosa FFM, Souza APR, Pretto JP, Moro MP, Oliveira LD. A experiência da maternidade e a dialogia mãe-filho com distúrbio de linguagem. Rev CEFAC. 2012;14(2):350-60. DOI: http://dx.doi.org/10.1590/S1516$\underline{18462010005000105 .}$

13. Carlesso JPP, Souza APR. Dialogia mãe-filho em contextos de depressão materna: revisão de literatura. Rev CEFAC. 2011;13(6):1119-26. DOI: http://dx.doi.org/10.1590/S1516-18462011005000085.

14. Flores MR, Beltrami L, Souza APR. O manhês e suas implicações para a constituição do sujeito na linguagem. Distúrb Comun. 2011;23(2):143-52.

15. Frizzo GB. A qualidade da interação pai-mãe-bebê em situação de depressão materna [dissertação]. Porto Alegre: Universidade Federal do Rio Grande do Sul; 2004.

16. Castro EK, Piccinini CA. A experiência da maternidade de mães de crianças com e sem doença crônica no segundo ano de vida. Est Psicol. 2004;9(1):89-99. DOI: http://dx.doi.org/10.1590/S1413-294X2004000100011.

17. Mahl FD, Mattiazzi AL, Angst OVM, Kessler TM, Biaggio EPV. Estudo do perfil sociodemográfico e psicossocial de mães de neonatos e seus conhecimentos sobre a Triagem Auditiva Neonatal. Mundo Saúde. 2013;37(1):35-43.

18. Hilu MRPB, Zeigelboim BS. O conhecimento, a valorização da triagem auditiva neonatal e a intervenção precoce da perda auditiva. Rev CEFAC. 2007;9(4):563-70. DOI: http://dx.doi.org/10.1590/S1516-18462007000400017.

19. Rangel SB, Ferrite S, Begrow DDV. Fatores que influenciam a não adesão ao retorno para a Triagem Auditiva Neonatal. Rev Baiana Saúde Pública. 2011;35(4):948-65.

20. Ribeiro FG, Mitre El. Avaliação do conhecimento sobre Triagem Auditiva Neonatal de pacientes no pós-parto imediato. Rev CEFAC. 2004;6(3):294-9.

21. Lewis DR, Marone SAM, Mendes BCA, Cruz OLM, Nóbrega M. Comitê multiprofissional em saúde auditiva: COMUSA. Braz J Otorhinolaryngol. 2010;76(1):121-8. DOI: http://dx.doi.org/10.1590/S1808-86942010000100020.

23. Gatto Cl, Tochetto TM. Deficiência auditiva infantil: implicações e soluções. Rev CEFAC. 2007;9(1):110-5. DOI: http:// dx.doi.org/10.1590/S1516-18462007000100014. 